normal to a point $(x, y, z)$ on the surface is given by an expression of the form

$$
d^{2}+A x^{2}+B y^{2}+\cdots,
$$

in which $A$ and $B$ are constants and the omitted terms are of the third order at least, and that when the given point is not a principal center of curvature the coefficients $A$ and $B$ do not vanish. If, however, the given point be a principal center of curvature, $A$ or $B$ will vanish and an examination of the sign of the non-vanishing coefficient will easily yield the first three theorems.

NEW YoRK, April 7, 1908.

\title{
ON THE SOLUTION OF ALGEBRAIC EQUATIONS IN INFINITE SERIES.
}

BY PROFESSOR P. A. LAMBERT.

(Read before the American Mathematical Society, April 25, 1908.)

I. Introduction.

The purpose of this paper is to present a general method for determining all the roots of any algebraic equation by means of infinite series. The method consists in forming three algebraic functions of $x$ from the given equation

$$
f(y)=0,
$$

(a) by introducing a factor $x$ into all the terms of (1) except the first and last ;

(b) by introducing a factor $x$ into all the terms of (1) except the first and second ;

(c) by introducing a factor $x$ into all the terms of (1) except the second and last.

These algebraic functions are expanded into power series in $x$ by Laplace's series. If in these power series $x$ is made unity, the resulting series, if convergent, determine the roots of the given equation. It will be shown that all the roots of the given equation can be expressed in infinite series derived either from the algebraic function formed in accordance with $(a)$, or from the two algebraic functions formed in accordance with $(b)$ and $(c)$. The method presupposes the solution of the two-term equation 


$$
a y^{n}+e=0 .
$$

In a paper entitled "New applications of Maclaurin's series in the solution of equations" read before the American Philosophical Society, April 3,1903, the author applied this method to numerical equations. After completing that paper, the author found in an extract of a letter from Cauchy to Coriolis, of January 29, 1837, published in the Comptes Rendus of the Paris Academy, an announcement of important results to be obtained by breaking up an equation into two parts and introducing as a factor into one part a parameter which is ultimately to be made unity. In a postscript Cauchy states that he discovered the advantage of making one part a binomial. The author has not been able to find the method sketched in this letter developed.

\section{The Three-Term Equation.}

From the three-term equation

$$
a y^{n}+b y^{k}+e=0
$$

in accordance with $(a),(b),(c)$ of Article I, are formed the three algebraic functions of $x$

$$
\begin{aligned}
& a y^{n}+b y^{k} x+e=0, \\
& a y^{n}+b y^{k}+e x=0, \\
& a y^{n} x+b y^{k}+e=0 .
\end{aligned}
$$

If equations (4), (5), (6) are written in the form

$$
\begin{aligned}
& y=\left[-\frac{e}{a}-x \frac{b}{a} y^{k}\right]^{\frac{1}{n}}, \\
& y=\left[-\frac{b}{a}-x \frac{e}{a} y^{-k}\right]^{\frac{1}{n-k}}, \\
& y=\left[-\frac{e}{b}-x \frac{a}{b} y^{n}\right]^{\frac{1}{b}},
\end{aligned}
$$

the algebraic functions $y$ can be expanded into power series in $x$ by means of Laplace's series, ${ }^{*}$ which asserts that if

* Mémoires de l'Académie de Sciences de Paris, 1777. 


$$
\begin{aligned}
y & =f[z+x \phi(y)], \\
F(y)=F\{f(z)\} & +x \phi\{f(z)\} \frac{d}{d z} F\{f(z)\} \\
& +\frac{x^{2}}{2 !} \frac{d}{d z}\left[\overline{\phi\{f(z)\}}^{2} \frac{d}{d z} F\{f(z)\}\right]+\cdots \\
& +\frac{x^{n}}{n !} \frac{d^{n-1}}{d z^{n-1}}\left[\overline{\phi\{f(z)\}}^{n} \frac{d}{d z} F\{f(z)\}\right]+\cdots
\end{aligned}
$$

If the algebraic function $y$ of equation (7) is expanded into a power series in $x$ by Laplace's series and $x$ is made unity in this series, and if $y_{0}$ denotes any one of the $n$ roots of the twoterm equation

there results $*$

$$
a y^{n}+e=0 \text {, }
$$

$$
\begin{gathered}
y=y_{0}+\frac{b}{n e} y_{0}^{1+k}+\frac{1+2 k-n}{2 !} \frac{b^{2}}{n^{2} e^{2}} y_{0}^{1+2 k} \\
+\frac{(1+3 k-n)(1+3 k-2 n)}{3 !} \frac{b^{3}}{n^{3} e^{3}} y_{0}^{1+3 k}+\cdots \\
+\frac{(1+s k-n)(1+s k-2 n) \cdots[1+s k-(s-1) n]}{s !} \frac{b^{s}}{n^{s} e^{s}} y_{0}^{1+s k}+\cdots
\end{gathered}
$$

If the first $n$ terms of series (12) are placed in a row in regular order, and the following $n$ terms are placed under the terms of this row in regular order, and so on indefinitely, the terms of series (12) will be arranged in $n$ partial infinite series composed respectively of the terms standing in each of the $n$ columns.

This rearrangement of terms is permissible inasmuch as it will appear subsequently that this series is used only when it is absolutely convergent.

The terms of the left-hand partial series are

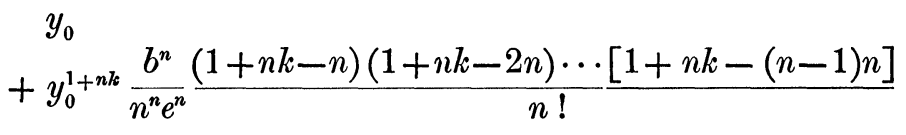

* Laplace's series is used only as a matter of convenience. The same expansion can be found by using Lagrange's series, Maclaurin's series, or the multinomial theorem by assuming $y=a_{0}+a_{1} x+a_{2} x^{2}+\cdots+a_{t} x^{t}+\cdots$ in equation (7). 


$$
\begin{aligned}
& (1+2 n k-n)(1+2 n k-n) \\
& +y_{0}^{1+2 n k} \frac{b^{2 n}}{n^{2 n} e^{2 n}} \frac{\cdots[1+2 n k-(2 n-1) n]}{(2 n) !} \\
& (13)+
\end{aligned}
$$

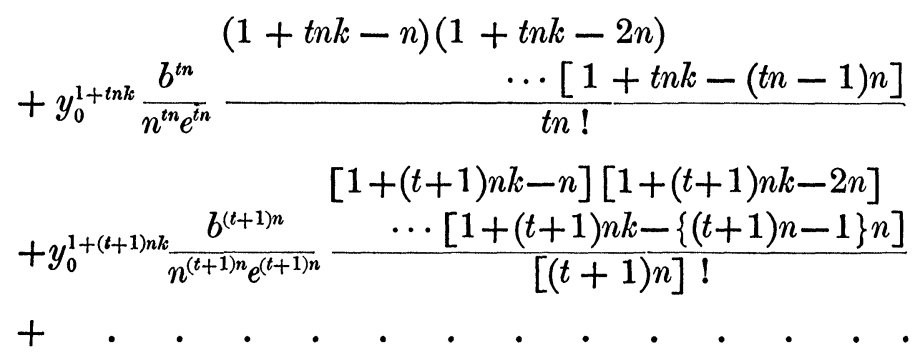

Cauchy's ratio test for convergency is applied to series (13). The ratio of the general term of (13) to the preceding term is

$$
\text { (14) } y_{0}^{n k} \frac{b^{n}}{n^{n} e^{n}} \frac{\begin{array}{c}
{[1+t n k+(k-1) n] \cdots[1+t n k][1-t(n-k) n]} \\
\cdots[1-t(n-k) n-(n-k-1) n]
\end{array}}{(t n+1)(t n+2) \cdots(t+1) n} \text {. }
$$

In each of the two groups of factors in the numerator of the last fraction in this ratio the successive factors differ by $-n$. The first group contains $k$ factors, the second group contains $n-k$ factors.

If the limiting value of this ratio (14) when $t$ becomes infinitely large is numerically less than unity, series (13) is absolutely convergent. This condition gives the inequality between absolute values

$$
\overline{e^{k}} \frac{b^{n}}{a^{k}} \frac{n^{n} e^{n}}{n^{k}}(n-k)^{n-k}<1,
$$

whence

$$
\left|\frac{b^{n}}{a^{k} e^{n-k}}\right|<\frac{n^{n}}{k^{k}(n-k)^{n-k}} .
$$

In like manner it is proved that the inequality (16) is the condition for the absolute convergence of each one of the remaining $n-1$ partial series into which the infinite series (12) has been broken up.

It follows that when condition (16) is satisfied the infinite series (12) is absolutely convergent. 
From equation (7), by means of Laplace's series (11), power series in $x$ can be found for $y^{n}$ and $y^{k}$. If in these power series $x$ is made unity, the resulting series for $y^{n}$ and $y^{k}$ are found to be absolutely convergent when condition (16) is satisfied. The values of $y^{n}$ and $y^{k}$ make equation (3) an identity. Therefore the value of $y$ in (12) is not only convergent when (16) is satisfied, but it actually represents a root of equation (3). In fact this expression for $y$ determines the $n$ roots of equation (3).

In like manner the series which express the roots of the three-term equation (3) derived from the power series obtained from equations (8) and (9) by Laplace's series are found to be absolutely convergent provided

$$
\left|\frac{b^{n}}{a^{k} e^{n-k}}\right|>\frac{n^{n}}{k^{k}(n-k)^{n-k}} .
$$

When condition (17) is satisfied the series obtained from (8) determines $n-k$, the series obtained from (9) determines $k$ roots of equation (3).

Raabe's convergency test* shows that the series which express the roots of equation (3) derived from equations (7), (8), (9) are all convergent for the limiting condition

$$
\left|\frac{b^{n}}{a^{k} e^{n-k}}\right|=\frac{n^{n}}{k^{k}(n-k)^{n-k}} .
$$

It follows that all the roots of the three-term equation (3) can always be expressed in infinite series derived either from equation (4) or from the two equations (5) and (6).

Infinite series for determining all the roots of the three-term equation, differing from series (12) only in form, are obtained by Nekrasoff, Mathematische Annalen, volume 29, by means of evaluating a definite integral, and by McClintock, in volume 17 of the American Journal of Mathematics, by means of his calculus of enlargement. Nekrasoff and McClintock both recognize that their series can be obtained by applying Lagrange's series.

Both investigators found convergency conditions corresponding to (16) and (17) by the methods used in this article. Condition (18) seems to be new.

* The infinite series $\Sigma_{n=1}^{n=\infty} u_{n}$ is convergent if

$$
\lim _{n=\infty}\left\{n\left(\frac{u_{n}}{u_{n+1}}-1\right)\right\}>1
$$

Zeitschrift für Mathematik und Physik, vol. 10 (1832). 
The results obtained by applying the method of Article I for finding infinite series determining the roots of equations of more than three terms in the following articles, so far as the author knows, are new.

\section{The Four-Term Equation.}

From the four-term equation

$$
a y^{n}+b y^{k}+c y^{l}+e=0
$$

in accordance with $(a),(b),(c)$ of Article I are formed the three equations

$$
\begin{aligned}
& a y^{n}+b y^{k} x+c y^{l} x+e=0, \\
& a y^{n}+b y^{k}+c y^{l} x+e x=0, \\
& a y^{n} x+b y^{k}+c y^{l} x+e=0,
\end{aligned}
$$

defining $y$ as algebraic functions of $x$.

The equations (20), (21), (22) may be written in the form

$$
\begin{aligned}
& y=\left[-\frac{e}{a}-x\left(\frac{b}{a} y^{k}+\frac{c}{a} y^{l}\right)\right]^{1 / n}, \\
& y=\left[-\frac{b}{a}-x\left(\frac{c}{a} y^{l-k}+\frac{e}{a} y^{-k}\right)\right]^{1 /(n-k)}, \\
& y=\left[-\frac{e}{b}-x\left(\frac{a}{b} y^{k}+\frac{c}{b} y^{l}\right)\right]^{1 / k} .
\end{aligned}
$$

If the $y$ of equation (23) is expanded into a power series in $x$ by Laplace's series, and after $x$ is made unity the terms of the resulting series are arranged in groups according to the ascending powers of

$$
\frac{c}{n e}\left(-\frac{e}{a}\right)^{l / n}
$$

it will be found that the terms of the first group constitute the series which expresses the roots of the three-term equation (3) provided condition (16) is satisfied, and that all the successive groups are convergent when condition (16) is satisfied.

It follows that the series which expresses the roots of equation (19) derived from equation (23) can be written 


$$
\begin{aligned}
y=X_{0}+X_{1} \frac{c}{n e}\left(-\frac{e}{a}\right)^{l / n}+ & X_{2} \frac{c^{2}}{n^{2} e^{2}}\left(-\frac{e}{a}\right)^{2 l / n} \\
& +X_{3} \frac{c^{3}}{n^{3} e^{3}}\left(-\frac{e}{a}\right)^{3 l / n}+\cdots
\end{aligned}
$$

where $X_{0}, X_{1}, X_{2}, X_{3}, X_{4}, \ldots$ represent convergent infinite series.

The series (27) is convergent provided

$$
\left|\frac{c}{n e}\left(-\frac{e}{a}\right)^{l / n}\right|<1 \text {. }
$$

Condition (28), when both members of the inequality are affected by the exponent $n$, becomes

The conditions

$$
\left|\frac{c^{n}}{a^{l} e^{n-l}}\right|<n^{n}
$$

$$
\left|\frac{b^{n}}{a^{k} e^{n-k}}\right| \leqq \frac{n^{n}}{k^{k}(n-k)^{n-k}}, \quad\left|\frac{c^{n}}{a^{l} e^{n-l}}\right|<n^{n}
$$

are therefore sufficient for the absolute convergence of the infinite series expressing the roots of the four-term equation (19) derived from equation (23).

In like manner it is found that the conditions sufficient for the absolute convergence of the infinite series expressing the roots of the four-term equation (19) derived from equations (24) and (25) are

$$
\begin{array}{ll}
\left|\frac{b^{n}}{a^{k} e^{n-k}}\right| \geqq \frac{n^{n}}{k^{k}(n-k)^{n-k}}, \quad\left|\frac{b^{n-l}}{a^{k-l} c^{n-k}}\right|>\frac{1}{(n-k)^{n-k}}, \\
\left|\frac{b^{n}}{a^{k} e^{n-k}}\right| \geqq \frac{n^{n}}{k^{k}(n-k)^{n-k}}, & \left|\frac{c^{k}}{b^{l} e^{k-l}}\right|<k^{k} .
\end{array}
$$

Either the first condition of (30) or the first condition of (31) and (32) must be satisfied. If it is possible to show that when the first condition of (30) or of (31) and (32) is satisfied the second inequalities can always be satisfied, the possibility of determining the roots of the four-term equation by means of infinite series is established.

The substitution

$$
y=z^{s},
$$


where $s$ is a positive integer, transforms the four-term equation (19) into the four-term equation

$$
a y^{n s}+b y^{k s}+c y^{l s}+e=0 .
$$

The convergency conditions for equation (34) corresponding to conditions (30), (31), (32) are

(35) $\left|\frac{b^{n}}{a^{k} e^{n-k}}\right| \leqq \frac{n^{n}}{k^{k}(n-k)^{n-k}}, \quad\left|\frac{c^{n}}{a^{l} e^{n-l}}\right|<s^{n} n^{n}$,

(36) $\left|\frac{b^{n}}{a^{k} e^{n-k}}\right| \geqq \frac{n^{n}}{k^{k}(n-k)^{n-k}}, \quad\left|\frac{b^{n-l}}{a^{k-l} c^{n-k}}\right|>\frac{1}{s^{n-k}(n-k)^{n-k}}$,

(37) $\left|\frac{b^{n}}{a^{k} e^{n-k}}\right| \geqq \frac{n^{n}}{k^{k}(n-k)^{n-k}}, \quad\left|\frac{e^{k}}{b^{l} e^{k-l}}\right|<s^{k} k^{k}$.

The value of $s$ can always be taken so large that the second inequality of (35) is satisfied, or that the second inequalities of (36) and (37) are satisfied simultaneously.

When conditions (35) are satisfied, all the roots of equation (34) are expressed by the infinite series derived from the equation

$$
a y^{n s}+b y^{k s} x+c y^{l s} x+e=0 ;
$$

when conditions (36) and (37) are satisfied, all the roots of equation (34) are expressed by the infinite series derived from the equations

$$
\begin{aligned}
& a y^{n s}+b y^{k s}+c y^{l s} x+e x=0, \\
& a y^{n s} x+b y^{k s}+c y^{l s} x+e=0 .
\end{aligned}
$$

It is therefore always possible to express all the roots of equation (34) in infinite series. The roots of equation (19) are found from the roots of equation (34) by substituting in (33).

IV. The Five-Term Equation.

From the five-term equation

$$
a y^{n}+b y^{k}+c y^{l}+d y^{m}+e=0,
$$

in accordance with $(a),(b),(c)$ of Article I, are formed the three equations 
1908.] SOLUTION OF ALGEBRAIC EQUATIONS.

$$
\begin{aligned}
& a y^{n}+b y^{k} x+c y^{l} x+d y^{m} x+e=0, \\
& a y^{n}+b y^{k}+c y^{l} x+d y^{m} x+e x=0, \\
& a y^{n} x+b y^{k}+c y^{l} x+d y^{m} x+e=0,
\end{aligned}
$$

defining $y$ as algebraic functions of $x$.

The equations (42), (43), (44) may be written in the form

$$
\begin{aligned}
& y=\left[-\frac{e}{a}-x\left(\frac{b}{a} y^{k}+\frac{c}{a} y^{l}+\frac{d}{a} y^{m}\right)\right]^{\frac{1}{n}}, \\
& y=\left[-\frac{b}{a}-x\left(\frac{a}{a} y^{l-k}+\frac{d}{a} y^{m-k}+\frac{e}{a} y^{-k}\right)\right]^{\frac{1}{n-k}}, \\
& y=\left[-\frac{e}{b}-x\left(\frac{a}{b} y^{n}+\frac{c}{b} y^{l}+\frac{d}{b} y^{m}\right)\right]^{\frac{1}{\bar{c}_{c}}} .
\end{aligned}
$$

If the $y$ of equation (45) is expanded into a power series in $x$ by Laplace's series and after $x$ is made unity in this power series the terms of the resulting series are arranged in groups according to the ascending powers of

$$
\frac{d}{n e}\left(-\frac{e}{a}\right)^{\frac{m}{n}}
$$

it will be found that the terms of the first group constitute the series which expresses the roots of the four-term equation (19) provided the conditions (30) are satisfied, and that all the successive groups are convergent when the conditions (30) are satisfied.

It follows that the series which expresses the roots of equation (41) derived from equation (42) can be written

$$
\begin{aligned}
y=Y_{0}+Y_{1} \frac{d}{n e}\left(-\frac{e}{a}\right)^{\frac{m}{n}}+Y_{2} & \frac{d^{2}}{n^{2} e^{2}}\left(-\frac{e}{a}\right)^{\frac{2 m}{n}} \\
& +Y_{3} \frac{d^{3}}{n^{3} e^{3}}\left(-\frac{e}{a}\right)^{\frac{3 m}{n}}+\cdots,
\end{aligned}
$$

where $Y_{0}, Y_{1}, Y_{2}, Y_{3}, \ldots$, represent convergent infinite series.

The series (49) is convergent provided

$$
\left|\frac{d}{n e}\left(-\frac{e}{a}\right)^{\frac{m}{n}}\right|<1
$$


Condition (50), when both members of the inequality are affected by the exponent $n$, becomes

The conditions

$$
\left|\frac{d^{n}}{a^{m} e^{n-m}}\right|<n^{n}
$$

$$
\left|\frac{b^{n}}{a^{k} e^{n-k}}\right| \leqq \frac{n^{n}}{k^{k}(n-k)^{n-k}}, \quad\left|\frac{c^{n}}{a^{l} e^{n-l}}\right|<n^{n}, \quad\left|\frac{d^{n}}{a^{m} e^{n-m}}\right|<n^{n}
$$

are therefore sufficient for the absolute convergence of the series expressing the roots of the five-term equation (41) derived from equation (42).

In like manner it is found that the conditions sufficient for the absolute convergence of the infinite series expressing the roots of the five-term equation (41) derived from equations (43) and (44) are

$$
\begin{gathered}
\left|\frac{b^{n}}{a^{k} e^{n-l k}}\right| \geqq \frac{n^{n}}{k^{k}(n-k)^{n-k}}, \quad\left|\frac{b^{n-l}}{a^{k-l} c^{n-k}}\right|>\frac{1}{(n-k)^{n-k}}, \\
\left|\frac{b^{n-m}}{a^{k-m} d^{n-k}}\right|>\frac{1}{(n-k)^{n-k}},
\end{gathered}
$$

$$
\left|\frac{b^{n}}{a^{k} e^{n-k}}\right| \geqq \frac{n^{n}}{k^{k}(n-k)^{n-k}}, \quad\left|\frac{c^{k}}{b^{l} e^{k-l}}\right|<k^{k}, \quad \frac{d^{k}}{b^{m} e^{k-m}}<k^{k} .
$$

Either the first condition of (52), or the first conditions of (53) and (54) must be satisfied.

The substitution

$$
y=z^{s}
$$

transforms the five-term equation (41) into the five-term equation

$$
a y^{n s}+b y^{k s}+c y^{l s}+d y^{m s}+e=0 .
$$

It is always possible to determine $s$ so that either the convergency conditions for equation (56) corresponding to (52), or those corresponding to (53) and (54) are satisfied. It is therefore always possible to determine the roots of equation (56) by infinite series. The roots of the five-term equation (41) are found from the roots of (56) by substituting in (55). 


\section{Conclusion.}

The method used to set up the convergency conditions for the infinite series expressing the roots of the four-term equation derived from the equations formed in accordance with $(a),(b)$ (c) of Article I when the convergency conditions of the threeterm equation are known, and to set up the convergency conditions for the five-term equation when the convergency conditions for the four-term equation are known, can be used to set up the convergency conditions for the $t$-term equation when the convergency conditions for the $(t-1)$-term equation are known.

In fact, the convergency conditions for an equation of any number of terms can be written mechanically.

$$
f(y)=0
$$

it is always possible to determine the $s$ of the substitution

$$
y=z^{s}
$$

so that the convergency conditions of the infinite series expressing the roots of the $t$-term equation

$$
f\left(z^{s}\right)=0
$$

derived from the equation formed from (59) in accordance with (a) of Article I, or derived from the two equations formed from (59) by (b) and (c) of Article I are satisfied. The roots of the $t$-term equation (57) are found from the roots of the equation (59) by substituting in (58).

It is therefore always possible to find all the roots of an algebraic equation by means of infinite series.

LEHIGH UNIVERSITY, April, 1908.

\section{THE DEDUCTION OF THE ELECTROSTATIC EQUATIONS BY THE CALCULUS OF VARIATIONS.}

BY DR. ARTHUR C. LUNN.

(Read before the Chicago Section of the American Mathematical Society, April 17, 1908.)

THE construction of a mathematical theory of classes of physical phenomena for which no detailed mechanical explanation 